\title{
1 Genome Editing Is Induced in a Binary Manner in Single Human Cells
}

$2{ }^{1}$ Gou Takahashi, ${ }^{1,2}$ Daiki Kondo, ${ }^{1,3}$ Minato Maeda, ${ }^{4}$ Yuji Morishita, and ${ }^{1,2}$ Yuichiro Miyaoka

$4 \quad{ }^{1}$ Regenerative Medicine Project, Tokyo Metropolitan Institute of Medical Science, Tokyo, Japan

$5{ }^{2}$ Graduate School of Medical and Dental Sciences, Tokyo Medical and Dental University, Tokyo,

6 Japan

7 '3epartment of Biological Sciences, Faculty of Science and Engineering, Chuo University,

8 Tokyo, Japan.

$9 \quad{ }^{4}$ On-chip Biotechnologies Co., Ltd., Tokyo, Japan.

11 Abstract

12 Even when precise nucleotide manipulations are intended, the outcomes of genome editing can

13 be diverse, often including random insertions and deletions. The combinations and frequencies

14 of these different outcomes in single cells are critical not only in the generation of genetically

15 modified cell lines but also in the evaluation of the clinical effects of genome editing therapies.

16 However, current methods only analyze cell populations, not single cells. Here, we utilized the

17 Single Particle isolation System (SPiS) for the efficient isolation of single cells to systematically analyze genome editing results in individual human cultured cells. As a result, we discovered that genome editing induction has a binary nature, that is, the target alleles of cells tend to be all edited or not edited at all. This study enhances our understanding of the induction mechanism of

21 genome editing and provides a new strategy to analyze genome editing outcomes in single cells. 
 \\ Introduction}

23 Genome editing allows us to manipulate genetic information in basically any type of cell, and has

24 been revolutionary in basic science, agriculture, and medicine ${ }^{1-3}$. Genome editing tools were

25 originally designed to cleave target sequences in the genome DNA in the cell, so that genetic

26 manipulations can be introduced into the genome via activated DNA repair pathways at the

27 target sites ${ }^{4}$. These DNA repair pathways include non-homologous end-joining (NHEJ) and

28 homology-directed repair (HDR) $)^{5}$. Each pathway gives distinct genome editing outcomes.

29 Although base editing and prime editing technologies do not require DNA double-strand breaks

30 to manipulate the genome DNA sequence, they can still never produce only one type of editing ${ }^{6-}$

319 . Despite significant progress in the prediction of genome editing outcomes ${ }^{10-13}$, it has been

32 impossible to produce a sole genetic manipulation. In other words, genome editing outcomes

33 are always mixtures of different modifications of DNA sequences, such as insertions or deletions

34 of different sizes and targeted recombination events. Therefore, for the application of genome

editing, it is important to precisely measure its outcomes.

Various types of techniques have been used to analyze genome editing outcomes ${ }^{14}$, including

sequencing-based methods (e.g., amplicon sequencing and TIDE ${ }^{15}$ ), denaturation-based

methods (e.g., T7E $1^{16}$ and single-stranded conformational polymorphism [SSCP] assays ${ }^{17}$ ), and

digital PCR-based methods ${ }^{18,19}$. However, all of these methods are designed to analyze cell

populations, not single cells. The combination of fluorescent reporter systems, such as traffic

light reporter ${ }^{20}$ and flow cytometry, can visualize genome editing results in individual cells, but

this setting cannot be applied to endogenous genes. To fully exploit the potential of genome

editing, it is critical to grasp the editing outcomes in individual cells. For example, in a

hypothetical situation where $50 \%$ of a population of diploid cells are WT/HDR heterozygotes and 
47 the two populations would - as a whole-have identical allelic frequencies of WT, HDR, and

NHEJ, while the cells would show a totally different composition (Fig. 1a). Therefore, there is a

strong demand for an efficient strategy to investigate genome editing outcomes in single cells.

50 The main reason why analyzing genome editing results in single cells has been so difficult is the

51 limitation in the number of target molecules (i.e., a diploid cell has only two copies of genomic

52 DNA). Even karyotypically abnormal cell lines only have several copies of target DNA

53

54

55

56

57

58

sequences per cell at most. This is in clear contrast to the recent advancement of the single cell transcriptome ${ }^{21}$, where a typical mammalian cell has $\sim 10^{5}$ mRNA molecules to analyze 22 . Therefore, it is extremely challenging to directly analyze the genomic DNA sequences in single cells. However, there are two clear advantages in the analysis of genomic DNA: one is that cells can replicate their own genome DNA as long as they are alive and proliferate; the other is that its sequence does not change, in contrast to the repertory of mRNA that changes dynamically depending on the cell state. Therefore, we decided to systematically isolate clones from a pool of genome edited cells. To accomplish this task, we utilized the Single Particle isolation System (SPiS) (On-chip Biotechnologies). The SPiS is an automated single cell dispenser that can gently and accurately dispense single cells in multi-well plates using image recognition technology to monitor the number of cells in aliquots ${ }^{23,24}$. The SPiS enabled us to isolate clones derived from genome edited cells with unprecedented efficiency. Our new pipeline to analyze genome editing based on the SPiS and findings from it will greatly contribute to improving the understanding of how genome editing occurs in the cell. 


\section{7 \\ Results}

\section{Measurement of the copy number of RBM20 in HEK293T cells}

First, we measured the copy number of our target gene, RBM20, per HEK293T cell to

comprehend genome editing outcomes in single cells, because cell lines often have abnormal

karyotypes. Therefore, we combined karyotyping and a comparative genomic hybridization

$(\mathrm{CGH})$ analysis to precisely estimate the copy number. We first karyotyped a total of 10

HEK293T cells, and found extensive chromosomal abnormalities (Fig. 1b). The most frequent

chromosomal number was three (Supplementary Table 1). We also found out that five cells each

had three and four chromosome 10s where RBM20 is located, respectively (Fig. 1b and

Supplementary Fig. 1a).

Next, we analyzed the genome DNA of HEK293T cells by a CGH analysis. We compared

HEK293T cells with WTC11 iPS cells that had been confirmed to be diploid ${ }^{19}$. The CGH analysis

also demonstrated the chromosomal abnormalities of HEK293T cells, as we revealed by

karyotyping (Fig. 1b, c). We detected peaks of the CGH signal corresponding to the

chromosome number in HEK293T cells (Fig. 1d). Because the most frequent chromosome

number was three (as determined by karyotyping), the most frequently observed peak of the

CGH signal corresponded to three copies. We were able to incrementally assign copy numbers

to these peaks to draw a line of fit between the relative CGH signal intensity and the copy

iPS cells around the RBM20 gene (Fig. 1f and Supplementary Fig. 1b, c) to this line of fit, we 
91 To systematically analyze the genome editing outcomes in individual cells, we established an

92 efficient pipeline to isolate cell clones that had gone through the genome editing process. Using

93 CRISPR-Cas9 and a single strand DNA donor in HEK293T cells, as we described previously ${ }^{18}$,

94 we introduced the RBM20 R636S mutation (c.1906C>A, chr.10), which causes inherited dilated

95 cardiomyopathy ${ }^{25}$ (Fig. 2a). We used pX458 (Addgene plasmid \#48138) to express EGFP via

96 the T2A peptide fused to Cas9 in combination with gRNA. Therefore, the expression of EGFP

97 guaranteed the expression of the CRISPR components in the same cell (Fig. 2b and

Supplementary Fig. 2a). To minimize damage in the sorting of these EGFP-positive cells, we

used a microfluidic cell sorter, On-chip Sort ${ }^{26}$. We confirmed that the sorting of EGFP-positive

cells successfully enriched genome-edited cells (Supplementary Fig. 2b). These sorted cells

101

were then subjected to the single cell isolation process using the SPiS, in which 384 cells were

individually plated into four 96-well plates. The SPiS aspirates diluted cell suspension into a

103

microtip and analyzes the images (taken by a CCD camera) of the contents. The camera takes

104

two images with a one-second interval; thus, the contents in the microtip go down by gravity.

The SPiS analyzes the two images to measure the velocity of precipitation and the size of the contents (Fig. 2c). By doing this, the SPiS can discriminate cells from other objects (e.g., cell debris and dust). The SPiS dispenses contents of a microtip into a culture dish, only when the outcomes of genome editing (Fig. 2b).

\section{Successful analysis of genome editing outcomes in single HEK293T cells}


117 substitution and insertions or deletions as "HDR" and "NHEJ" events, respectively, while

118 combinations of both in the same allele were defined as "HDR+NHEJ" events (Fig. 2a and

119 Supplementary Fig. 3b). HDR+NHEJ events occurred at a relatively low frequency in

120 comparison to NHEJ, and the HDR frequency was even lower (Fig. 3a). Based on these

121 HEK293T cell clones with edited alleles, we were able to estimate the overall allelic frequencies

122 of each genome editing event (i.e., WT, NHEJ, HDR, and HDR+NHEJ) (Fig. 3b). Thus, our

123 SPiS-based approach enables us to systematically analyze genome editing outcomes in

124 individual cells.

125

126 Binary induction of genome editing in single cells

127 One notable feature in our results was that roughly half of the cells had all their target RBM20

128 alleles edited by $\operatorname{NHEJ}(47 / 128,62 / 109$, and 44/93, respectively). On the other hand, there were 129 quite a few cells that remained completely unedited (49/128, 26/109, and 24/93, respectively), 130 although these cells had expressed Cas9 and gRNA (Fig. 3a). These observations were quite 131 surprising considering the fact that these cells have three or four copies of RBM20 on average 132 (Fig. 1).

133 To address whether this trend is significant, we mathematically calculated the number of

134 HEK293T cell clones with different genome editing outcomes assuming that these events 135 occurred randomly. Because the copy number of RBM20 was 3.54 (Fig. 1), we built two models 136 with three or four copies of RBM20 (Fig. 3c and Supplementary Fig. 4). In these models, we calculated how many cells with three or four copies of RBM20 should have specific genotypes

138 based on the overall frequencies of WT, NHEJ, HDR, and HDR+NHEJ alleles. We compared the proportions of clones that are expected to be unedited to remain as WT or fully edited by NHEJ 
141 of actually isolated clones were significantly higher than those based on the models for both WT

142 and full NHEJ clones (Fig. 3d). In addition, among 330 clones, we observed one clone in which

143 all target alleles were modified by HDR (Fig. 3a). No such clone was expected in the

144 mathematical models (Fig. 3c and Supplementary Fig. 4). These results suggested that genome

145 editing occurs in a binary manner, in which cells show either no editing at all or full editing.

147 HypaCas9 induces HDR more efficiently than Cas9 in single cells

148 Because the frequency of HDR was so low with Cas9, we were not able to investigate how HDR

149 is induced in single cells (Fig. 3). HDR is often more desirable than NHEJ, as HDR can

150 introduce precise manipulation of the DNA sequence. Therefore, we decided to apply our SPiS-

151 based analysis to genome editing conditions that are more favorable for the induction of HDR.

152 We found that Cas9 with improved proof-reading (e.g., HypaCas9 ${ }^{28}$, Cas9-HF1 ${ }^{29}$, and

153 eSpCas9 ${ }^{30}$ ) induced more HDR than Cas9 in our previous cell population-based assay ${ }^{31}$; thus,

154 we targeted the same RBM20 R636S mutation in HEK293T cells using HypaCas9, and

155 conducted the SPiS-based single cells assay three times. We confirmed that HypaCas9

156 produced more clones with HDR than Cas9 (Fig. 4a). We calculated the allelic frequencies of

157 WT, NHEJ, HDR, and HDR+NHEJ (Fig. 4b), and compared these between Cas9 and

158 HypaCas9. We found that the HDR allelic frequency was higher with HypaCas9 than with Cas9;

159 however, the WT, NHEJ, and HDR+NHEJ allelic frequencies were comparable (Fig. 4c). Based

160 on these overall allelic frequencies, we built mathematical models of HEK293T cell clones with

161 genome editing by HypaCas9 in the same way as for Cas9 (Fig. 4d and Supplementary Fig. 5).

162 The binary manner of genome editing induction was also observed with HypaCas9, as the WT

163 and full NHEJ clone proportions were significantly higher than the mathematical models (Fig.

1644 4). Moreover, binary induction was also observed in HDR. Among 422 clones, we isolated a

165 total of six clones with all alleles edited by HDR (Fig. 4a). Because the overall frequency of HDR 
was $5.89 \%$ (Fig. 4c), no such clone was expected to be isolated if genome editing events

167 occurred randomly in single cells ( 1 out of 4894 cells would be expected to have having three

168 HDR alleles and 1 out of 83088 cells would be expected to have four HDR alleles). Thus, we

169 confirmed the binary induction of genome editing, even with HypaCas9, which induces more

170 HDR than Cas9. The binary mode was observed in the induction of both HDR and NHEJ.

HDR is more often accompanied by NHEJ than WT

We noticed that clones with HDR often had NHEJ alleles unless cells were fully edited by HDR.

174 Therefore, we calculated the proportions of clone that would be expected to have only HDR and

175 WT alleles, as these clones represent partial editing by HDR. Even though $2.5-3.5 \%$ of the cells

177 further investigated the expected and observed the proportions of clones with both HDR and

NHEJ events, including any clones with the HDR+NHEJ alleles, as they represent HDR and

NHEJ events induced in the same alleles. As we expected, the proportions of clones with both

HDR and NHEJ events that were observed and those that were predicted by the models were NHEJ are often induced together in single cells (Fig. 4f).

\section{Genome editing is also induced in a binary manner in ATP7B and GRN}

We next addressed whether the observed binary fashion of genome editing stands in other 
191 amplicon sequencing to quantify the frequency of genome editing (Fig. 5a-e). To build models

192 for genome editing of ATP7B and GRN, we quantified the copy numbers of these genes, which

193 were determined to be 2.88 for ATP7B and 3.54 for GRN (Fig. 1 and Supplementary Fig. 6c, d).

194 We then calculated the expected clone numbers with the assumption that genome editing

195 events occurred randomly in the cells to build models for genome editing of ATP7B and GRN

196 (Supplementary Fig. 7a, b), and compared them to the observed results. Similar to the RBM20

197 R636S mutagenesis, the number of clones in which all target alleles were edited by NHEJ or not

198 edited at all was significantly higher in comparison to the models (Fig. 5b, e). We also isolated

199 clones in which all target alleles were edited by HDR (8/280 and 2/276 for ATP7B and GRN,

200 respectively) (Fig. 5a, d). This was particularly noteworthy for GRN, as the overall efficiency of

201 HDR induction was only $1.02 \%$ (Fig. 5d). Collectively, these results demonstrate that the binary

202 nature of genome editing is not restricted to a specific target site. Moreover, we did not isolate

203 any clones with partial HDR editing in ATP7B or GRN; however, HDR and NHEJ were often

204 induced together in single cells, similarly to in RBM20 (Figs. 5a, c, d).

205

Binary genome editing is also prominent in HeLa cells

207 We further addressed whether the binary induction of genome editing occurs in other cell types.

208 For this purpose, we introduced the RBM20 R636S (c.1906C>A), ATP7B R778L (c.2333G>T),

209 and GRN R493X (c.1477C>T) mutations into HeLa cells in the same way as for HEK293T cells.

210 We were able to apply our SPiS-based system to isolated HeLa cell clones that had gone

211 through the genome editing process, although HeLa cells exhibited lower cell survival in

212 comparison to HEK293T cells (Fig. 6a-g). We measured the copy numbers of these target

213 genes by combining the karyotyping and CGH analyses in HeLa cells (Supplementary Fig. 8 and

214 Supplementary Table 2). Based on these copy numbers, mathematical models of clones with

215 different genotypes were built in the same way as for HEK293T cells (Supplementary Fig. 9). 
We found that the observed proportions of clones that were unedited or fully edited by NHEJ

217 were significantly higher than in the calculated models based on the overall allelic frequencies in

218 all three genes (Fig. 6b, d, g). The HDR frequency was higher in ATP7B than in RBM20 or GRN

219 in HeLa cells, and we isolated 10 clones fully edited by HDR out of 234 clones (Fig. 6c). In

220 ATP7B, the proportion of clones with partial editing by HDR was significantly lower in

221 comparison to the model, while that of clones with HDR accompanied by NHEJ was comparable

222 to the model (Fig. 6e). These results indicate that the binary nature of genome editing induction

223 is shared between HeLa cells and HEK293T cells.

225 Binary genome editing is less evident in PC9 cells

226 Finally, we investigated the binary nature of genome editing in another cell line, PC9 cells. We

227 introduced the same three mutations into PC9 cells and conducted our SPiS-based analysis

228 (Fig. 7a-f). We noticed that the genome editing efficiency in PC9 cells was generally lower than

229 that in HEK293T cells or HeLa cells, and that HDR was barely induced (Fig. 7a, c, e). We

230 measured the copy numbers of the target genes in PC9 cells (Supplementary Fig. 10 and

231 Supplementary Table 3). Based on these copy numbers, the mathematical models of clones

232 with different genotypes were built for PC9 cells (Supplementary Fig. 11). We compared the

233 proportion of clones that were unedited or fully edited by NHEJ between the observations and

234 the models. We found that the trend regarding the binary induction of genome editing was

235 noticeable but less evident, as the proportions of clones fully edited by NHEJ in RBM20 and

236 clones unedited in ATP7B were the only significant differences between the observed clones

237 and the models (Fig. 7b, d, f). Therefore, binary induction is a general feature of genome editing;

238 however, the extent of binary induction can vary in different contexts. 
241 Most of the current methods used to analyze genome editing outcomes deal with cell

242 populations. In this study, we were able to isolate single cell clones with an unprecedented

243 efficiency by the SPiS. The SPiS not only increased the number of clones that we were able to

244 isolate, but also ensured that the isolated clones were derived from single cells based on the

245 image analysis, which avoided plating multiple cells together. This original system allowed us to

246 investigate genome editing outcomes in single cells.

247 We were surprised to observe that most HEK293T cells were either unedited WT or had all

248 targeted alleles fully manipulated by Cas9. The number of partially edited cells that had both

249 unedited WT alleles and edited alleles was limited (Fig. 3). We previously reported that Cas9

250 variants with enhanced proof-reading are more efficient at inducing HDR than regular Cas $9^{31}$.

251 We confirmed that HypaCas9 produced more HDR in single cells than Cas9, and found that

252 HypaCas9 also induced genome editing in a binary fashion (Fig. 4d). This trend was shared

253 among all RBM20, ATP7B, and GRN genes in both HEK293T cells and HeLa cells, and to a

254 lesser extent in PC9 cells (Figs. 4-7). Because we sorted cells that expressed EGFP together

255 with Cas9 and gRNA, these WT clones escaped from DNA cleavage by Cas9, or precisely

256 repaired the genomic DNA after DNA cleavage. We speculate that each cell has an intracellular

257 environment that is favorable for a particular genome editing outcome, so that all target alleles in

258 one cell tend to have the same fate. The average copy numbers of RBM20, ATP7B, and GRN in

259 HEK293T cells, HeLa cells, and PC9 cells were 2.88-3.71 (Fig. 1 and Supplementary Figs. 1, 8,

260 and 10). The fact that some cells have full HDR or HDR+NHEJ events in roughly 3 to 4 copies

261 despite the much lower overall frequencies also suggests that target alleles in one cell tend to

262 have the same fate (Figs. 4-6). This binary nature of genome editing can be beneficial if

263 homozygous mutagenesis is necessary. Indeed, we previously reported the isolation of a human

264 iPS cell line with a homozygous PHOX2B Y14X mutation generated by TALENs, even though

265 the overall induction efficiency of HDR was just approximately $1 \%{ }^{19}$. At the same time, however, 
it makes heterozygous mutagenesis extremely difficult, as we observed that HDR was more

267 often accompanied by NHEJ rather than unedited WT alleles (Figs. 4-6).

268 At this point, however, the factors that determine which DNA repair pathway a single cell takes

269 remain unknown. One possibility is that the cells respond differently to Cas9 cleavage at

270 different points in the cell cycle. It is known that the frequency of HDR and NHEJ increases in

271 the S/G2 and G1 phases, respectively ${ }^{34,35}$. Therefore, it would be interesting to address whether

272 the cell cycle also influences this binary choice between full editing and no editing in single cells.

273 PC9 cells showed a much lower efficiency of overall induction of genome editing than HEK293T

274 cells and HeLa cells (Fig. 7). The binary fashion of induction of genome editing was also less

275 evident in PC9 cells than in HEK293T cells and HeLa cells. This could be due to the difference

276 in the expression of HypaCas9 protein. We observed a relatively low gene transduction

277 efficiency and low expression level in PC9 cells in comparison to the other two cell lines based

278 on the expression level of EGFP, which was co-expressed with Cas9 via the T2-peptide

279 (Supplementary Fig. 12). Therefore, it is possible that certain levels of Cas9 expression and

280 activity are necessary for the binary induction of genome editing. Another possibility is that

281 different DNA repair pathways are active in different cell types to yield different genome editing

282 outcomes. In PC9 cells, HDR was barely induced even with HypaCas9, which could be because

283 the HDR pathway is not very active in PC9 cells. An investigation of the active DNA repair

284 pathways in these cell lines would be an interesting way to address the observed differences in

285 genome editing outcomes.

286 We are currently applying the same analytic procedure to other cell types to further address the

287 generality of our findings. Our target cells types include human iPS cells, because we are highly

288 interested in the genome editing outcomes of normal diploid human cells. However, the biggest

289 challenge is the low survival rate of iPS cells, especially in single cell culture ${ }^{36 ;}$ thus, we are

290 optimizing the culture conditions. We can also apply different transduction methods, including 
291 lipofection, electroporation of plasmids and RNPs, and viral infection. We will test these various

292 parameters by our SPiS-based strategy to figure out general characteristics of genome editing

293 outcomes in single cells, and also best conditions to induce specific types of genome editing.

294 Our study reveals the previously unknown but fundamental features of genome editing in single

295 cells including the "binary nature" of genome editing induction. This was only possible with the

296 SPiS. Our findings contribute to the better understanding of the underlying mechanism of

297 induction of genome editing. Moreover, NHEJ often results in gene disruption; thus, the gene

298 functions in a cell may be lost in clones fully edited by NHEJ. This can be very important in

299 genome editing therapy that is dependent on HDR, as by-products of NHEJ might hamper the

300 therapy if the function of a certain gene is completely lost in some cells. Therefore, the analysis

301 of genome editing results in single cells is critical in precise evaluation of therapeutic effects. Our

302 SPiS also provides researchers with a versatile platform to study genome editing in single cells. 
Methods

\section{Statistical analyses}

305 The transfection and single cell dispensing experiments were performed in triplicate (three 306 biological replicates). Clones isolated by the SPiS that showed no amplification of target sites by 307 PCR or no successful alignments of amplicon sequencing reads by CRISPResso $2^{27}$ were 308 excluded from the analyses. Statistical significance between two groups was assessed by a 309 non-paired two-tailed Student's t-test.

\section{Plasmids and oligonucleotides for transfection}

We generated a vector that co-expresses EGFP and HypaCas9 (pX458-HypaCas9), based on pX458 (Addgene \#48138). The single strand DNA donors were all $60 \mathrm{nt}$ and had point mutations

314 in the middle of them. The sequences of the single strand DNA donors and gRNAs that were 315 used in this study are summarized in Supplementary Tables S4 and S5, respectively. All the single strand DNA donors and oligonucleotides for gRNA cloning, which were purified by standard desalting, were purchased from FASMAC, Japan.

\section{Calculation of the copy number of target genes by karyotyping and CGH analysis}

The karyotyping analysis of the cell lines was performed by Nihon Gene Research Laboratories. Karyotypes were analyzed in 10 cells for each cell line. The CGH assays were performed using the SurePrint G3 Human CGH Microarray Kit (Agilent) and the genomic DNA of WTC11 cells as a reference of the diploid cells. The genomic DNA of the cell lines used in this study for the CGH analysis was extracted using the DNeasy Blood and Tissue Kit (QIAGEN) according to the manufacturers' instructions. The CGH analysis gave peaks of the frequently observed signal intensity, which correspond to the number of chromosomes. Therefore, the chromosomal 
328 peak in the $\mathrm{CGH}$ array (for example, because the most frequently observed chromosomal

329 number was 3 by karyotyping of HEK293T cells, the most frequent peak of the signal intensity in

330 the $\mathrm{CGH}$ assay corresponded to 3 copies). Then, we were able to incrementally assign copy

331 numbers to the peaks in the $\mathrm{CGH}$ assay around the highest peak to draw a line of fit between

332 the copy number and the CGH peak values. The precise copy number of the target gene was

333 calculated by this line of fit and the signal intensity around the target genes measured by the

334 CGH assay.

335

336 Cell culture, transfection, and cell sorting

337 HEK293T cells and HeLa cells were maintained in Dulbecco's modified Eagle medium (DMEM) 338 with high glucose, sodium pyruvate, and L-glutamine (Thermo Fisher Scientific) supplemented 339 with 10\% fetal bovine serum (FBS, Thermo Fisher Scientific) and 1\% Penicillin-Streptomycin (P/S, 340 Sigma-Aldrich). PC9 cells were maintained in Roswell Park Memorial Institute (RPMI) medium 341 with L-glutamine (Nacalai Tesque) supplemented with 10\% FBS (Thermo Fisher Scientific) and $3421 \% \mathrm{P} / \mathrm{S}$. For transfection, $2 \times 10^{5}$ cells were plated in a well of a $12-$ well plate coated with $80 \mu \mathrm{g} / \mathrm{ml}$ 343 Growth Factor Reduced Matrigel Basement Membrane Matrix (BD Biosciences). One day later, 344 the cells were transfected with 720 ng of pX458 or pX458-HypaCas9 with a gRNA targeting 345 RBM20, ATP7B, or GRN, and 80 ng of oligonucleotide donor DNA using 2.4 $\mu$ l of Lipofectamine 3462000 (Thermo Fisher Scientific) for HEK293T cells and HeLa cells, and $2.0 \mu$ l of Lipofectamine 3473000 (Thermo Fisher Scientific) for PC9 cells, respectively, per well, according to the 348 manufacturers' instructions. After 24 hours, cells were detached by $0.25 \%$ Trypsin-EDTA (Thermo 349 Fisher Scientific) and EGFP-positive cells were sorted using On-ship Sort (On-chip 350 Biotechnologies). The isolated EGFP-positive cells were dispensed one by one into Matrigel351 coated 96-well plates using the On-chip Single Particle isolation System (SPiS) (Fig. 2c). 352 Subsequently, $100 \mu \mathrm{l} /$ well of conditioned medium was added to the plates with dispended cells. 
353 One week after single cell dispensing by SPiS, the medium was changed. Two weeks after

354 dispensing, for HEK293T cells and HeLa cells, the surviving colonies were first detached by

355 Trypsin-EDTA, then the cells were resuspended in DMEM supplemented with $20 \%$ FBS and $1 \%$

356 P/S to evenly re-distribute the cells within the wells of a 96-well plate. The cells were cultured in

357 this media until they reached confluence.

\section{Preparation of multiplexed amplicon sequencing library}

360 Genomic DNA was extracted from the cells in 96-well plates as described previously ${ }^{31}$. The DNA

was resuspended in $30 \mu \mathrm{l} /$ well of water. Targeted sites were amplified by the first PCR round

using primers with homology to the region of interest and the Illumina forward and reverse

forward and reverse primer, $1.0 \mu \mathrm{l}$ of genomic DNA, $2.0 \mu \mathrm{l}$ of $2 \mathrm{mM}$ dNTPs, $5.0 \mu \mathrm{l}$ of $2 \times \mathrm{PCR}$

Buffer KOD FX (Toyobo), $0.1 \mu$ l of KOD FX enzyme (Toyobo), $2 \mu$ l of betaine (Fuji Film), and 0.3

$\mu$ of water. The thermal cycling settings were as follows: $95^{\circ} \mathrm{C}$ for $2 \mathrm{~min}$, then 30 cycles of

$\left(95^{\circ} \mathrm{C}\right.$ for $30 \mathrm{sec}, 60^{\circ} \mathrm{C}$ for $30 \mathrm{sec}$, and $72^{\circ} \mathrm{C}$ for $1 \mathrm{~min}$ ), followed by a final $72^{\circ} \mathrm{C}$ extension for

3 min. The first PCR products were diluted by adding $90 \mu$ of water. Then, the DNA barcodes

and illumina adaptors were added to the amplicons in the second PCR round, which consisted

of $0.3 \mu \mathrm{l}$ each of $100 \mu \mathrm{M}$ unique forward and reverse index barcoding primers ${ }^{37}$ (Supplementary

table 7), $0.5 \mu \mathrm{l}$ of the diluted first PCR product, $2 \mu \mathrm{l}$ of $2 \mathrm{mM}$ dNTPs, $5.0 \mu \mathrm{l}$ of $2 \times$ PCR Buffer

KOD FX, $0.1 \mu$ l of KOD FX enzyme, and $1.8 \mu$ l of water. The thermal cycling settings were as

follows: $98^{\circ} \mathrm{C}$ for $3 \mathrm{~min}$, then 30 cycles of $\left(98^{\circ} \mathrm{C}\right.$ for $30 \mathrm{sec}, 57^{\circ} \mathrm{C}$ for $30 \mathrm{sec}$, and $72^{\circ} \mathrm{C}$ for

$3741 \mathrm{~min}$ ), followed by a final $72^{\circ} \mathrm{C}$ extension for $3 \mathrm{~min}$. The PCR products were electrophoresed in

$3752 \%$ agarose gel. NucleoSpin Gel and PCR Clean-up Midi kit (MACHEREY-NAGEL) were used

376 to extract the pooled PCR products from 24 samples in $200 \mu$ l of water. The DNA concentrations

377 of these library mixtures of 24 samples were quantified using the GenNext NGS Library 
378 Quantification Kit (Toyobo). All libraries were then mixed in equimolar amounts, 20\% PhiX

379 Control v3 (illumina) was added for amplicon sequencing. Sequencing was performed with

380 MiSeq (Illumina) using the Miseq v2 reagent kit (Illumina) or Miseq Reagent kit v2 Nano

381 (illumina) according to the manufacturer's instructions.

382

383

\section{Amplicon sequencing data analysis}

384 Fastq files generated by MiSeq were imported into the CLC Genomics Workbench 21

385 (QIAGEN). Adapter sequences were removed and demultiplexed using the DNA Index. The data were then analyzed by CRISPResso2 ${ }^{27}$ (https://github.com/pinellolab/CRISPResso2) in the

CRISPResso Batch mode. CRISPResso2 was installed as recommended using a Docker

containerization system. The commands for the CRISPResso2 analysis used in this study are

listed in Supplementary Table 8 . Alleles with $\leq 5 \%$ frequency were excluded from the analysis as they were expected to be either sequence errors or very minor populations generated during cell

proliferation from single cells. In the CRISPResso2 analysis, any alleles with deletions spanning the nucleotides for single nucleotide substitutions were characterized as "ambiguous".

Therefore, we classified these "ambiguous" alleles as NHEJ alleles in this study.

\section{Creating a model in which alleles were evenly distributed}

Models assuming that the WT, NHEJ, HDR, and HDR+NHEJ alleles were randomly induced in

all target alleles were built by distributing these edited alleles at their observed overall

frequencies. The number of target alleles per cell was calculated by karyotyping and a CGH analysis, as described above. For example, when ATP7B was targeted in HEK293T cells, one 
$403(100 \times(0.4 \times 0.3 \times 0.2)=2.4$, rounded up to 2$)$. We calculated the expected clone numbers of all the

404 possible combinations of WT, NHEJ, HDR, and HDR+NHEJ alleles, and combined them to build 405 the models.

406

407

\section{Data availability}

408 Raw data of multiplexed amplicon sequencing in this study are available in the DDBJ Sequence 409 Read Archive under the accession numbers DRA013570.

\section{Acknowledgements}

412 We thank Drs. Y. Fujimura and K. Takeda (On-chip Biotechnologies) for their helpful

413 discussions.

414 This work was supported by JSPS Grant-in-Aid for Challenging Research (Pioneering)

415 20K21409, Grant-in-Aid for Scientific Research (B) 20H03442, Interstellar Initiative from AMED 416 (20jm0610032h0001), Takeda Science Foundation Medical Research Grant, Sumitomo

417 Foundation Grant For Basic Science Research Projects, Ichiro Kanehara Foundation Grant to 418 Y.M., and a JSPS Grant-in-Aid for Early-Career Scientists (18K15054) to G.T.

\section{Author contributions}

421 G.T. and Y.Miyaoka conceived the idea of this study and designed the experiments. G.T., D.K., 422 and M.M. isolated genome edited HEK293T cell clones with technical help from Y.Morishita.

423 D.K. and M.M. prepared libraries for amplicon sequencing. G.T., D.K., M.M., and Y.Miyaoka 424 analyzed the results. Y.Miyaoka supervised the project. G.T. and Y.Miyaoka wrote the 425 manuscript with help from all of the authors. 
428 Y.Morishita is an employee of On-chip Biotechnologies Co., Ltd.

429

\section{Materials and correspondence}

431 Correspondence and requests for materials should be addressed to Yuichiro Miyaoka.

References

434 1. Cong, L. et al. Multiplex genome engineering using CRISPR/Cas systems. Science 339, 819-823 (2013).

2. Jinek, M. et al. A programmable dual-RNA-guided DNA endonuclease in adaptive bacterial immunity. Science 337, 816-821 (2012).

3. Mali, P. et al. RNA-guided human genome engineering via Cas9. Science 339, 823-826 (2013).

4. Gaj, T., Gersbach, C. A. \& Barbas, C. F. ZFN, TALEN, and CRISPR/Cas-based methods for genome engineering. Trends Biotechnol. 31, 397-405 (2013).

5. Yeh, C. D., Richardson, C. D. \& Corn, J. E. Advances in genome editing through control of DNA repair pathways. Nat. Cell Biol. 21, 1468-1478 (2019).

6. Nishida, K. et al. Targeted nucleotide editing using hybrid prokaryotic and vertebrate adaptive immune systems. Science 353, (2016).

446 7. Gaudelli, N. M. et al. Programmable base editing of $A \cdot T$ to $G \cdot C$ in genomic DNA without $447 \quad$ DNA cleavage. Nature 551, 464-471 (2017).

448 8. Komor, A. C., Kim, Y. B., Packer, M. S., Zuris, J. A. \& Liu, D. R. Programmable editing of a target base in genomic DNA without double-stranded DNA cleavage. Nature 533, 420-424 (2016). 
453 10. Abadi, S., Yan, W. X., Amar, D. \& Mayrose, I. A machine learning approach for predicting CRISPR-Cas9 cleavage efficiencies and patterns underlying its mechanism of action. PLoS Comput. Biol. 13, e1005807 (2017).

11. Arbab, M. et al. Determinants of Base Editing Outcomes from Target Library Analysis and Machine Learning. Cell 182, 463-480.e30 (2020).

12. Marquart, K. F. et al. Predicting base editing outcomes with an attention-based deep learning algorithm trained on high-throughput target library screens. Nat. Commun. 12, 5114 (2021).

13. Song, M. et al. Sequence-specific prediction of the efficiencies of adenine and cytosine base editors. Nat. Biotechnol. 38, 1037-1043 (2020).

14. Germini, D. et al. A comparison of techniques to evaluate the effectiveness of genome editing. Trends Biotechnol. 36, 147-159 (2018).

15. Brinkman, E. K., Chen, T., Amendola, M. \& van Steensel, B. Easy quantitative assessment of genome editing by sequence trace decomposition. Nucleic Acids Res. 42, e168 (2014).

16. Mashal, R. D., Koontz, J. \& Sklar, J. Detection of mutations by cleavage of DNA

17. Inazuka, M., Wenz, H. M., Sakabe, M., Tahira, T. \& Hayashi, K. A streamlined mutation detection system: multicolor post-PCR fluorescence labeling and single-strand conformational polymorphism analysis by capillary electrophoresis. Genome Res. 7, 1094-

18. Miyaoka, Y. et al. Systematic quantification of HDR and NHEJ reveals effects of locus, nuclease, and cell type on genome-editing. Sci. Rep. 6, 23549 (2016).

19. Miyaoka, Y. et al. Isolation of single-base genome-edited human iPS cells without antibiotic selection. Nat. Methods 11, 291-293 (2014). 
Nat. Methods 8, 671-676 (2011).

21. Aldridge, S. \& Teichmann, S. A. Single cell transcriptomics comes of age. Nat. Commun. 11, 4307 (2020).

481

482

483

484

485

486

487

488

489

490

491

492

493

494

495

496

497

498

499

500

501

22. Islam, S. et al. Characterization of the single-cell transcriptional landscape by highly multiplex RNA-seq. Genome Res. 21, 1160-1167 (2011).

23. Koike, S. The risk of unintentional propagation of poliovirus can be minimized by using human cell lines lacking the functional CD155 gene. Microbiol. Immunol. 64, 835-839 (2020).

24. Saito, K. et al. Microdroplet-based system for culturing of environmental microorganisms using FNAP-sort. Sci. Rep. 11, 9506 (2021).

25. Brauch, K. M. et al. Mutations in ribonucleic acid binding protein gene cause familial dilated cardiomyopathy. J. Am. Coll. Cardiol. 54, 930-941 (2009).

26. Takeda, K., Fujimura, Y. \& Jimma, F. Development of a new compact cytometer using a disposable microfluidic chip for contamination-free and biosafety measurement. (2011).

27. Clement, K. et al. CRISPResso2 provides accurate and rapid genome editing sequence analysis. Nat. Biotechnol. 37, 224-226 (2019).

28. Chen, J. S. et al. Enhanced proofreading governs CRISPR-Cas9 targeting accuracy. BioRxiv (2017). doi:10.1101/160036

29. Kleinstiver, B. P. et al. High-fidelity CRISPR-Cas9 nucleases with no detectable genomewide off-target effects. Nature 529, 490-495 (2016).

30. Slaymaker, I. M. et al. Rationally engineered Cas9 nucleases with improved specificity. Science 351, 84-88 (2016).

31. Kato-Inui, T., Takahashi, G., Hsu, S. \& Miyaoka, Y. Clustered regularly interspaced short palindromic repeats (CRISPR)/CRISPR-associated protein 9 with improved proof-reading enhances homology-directed repair. Nucleic Acids Res. 46, 4677-4688 (2018). 
503 32. Ferenci, P. Regional distribution of mutations of the ATP7B gene in patients with Wilson

504 disease: impact on genetic testing. Hum. Genet. 120, 151-159 (2006).

505 33. Chen-Plotkin, A. S. et al. Genetic and clinical features of progranulin-associated

506 frontotemporal lobar degeneration. Arch. Neurol. 68, 488-497 (2011).

507 34. Lin, S., Staahl, B. T., Alla, R. K. \& Doudna, J. A. Enhanced homology-directed human

508 genome engineering by controlled timing of CRISPR/Cas9 delivery. Elife 3, e04766 (2014).

509 35. Wienert, B. et al. Timed inhibition of CDC7 increases CRISPR-Cas9 mediated templated

510 repair. Nat. Commun. 11, 2109 (2020).

511 36. Watanabe, K. et al. A ROCK inhibitor permits survival of dissociated human embryonic stem

512 cells. Nat. Biotechnol. 25, 681-686 (2007).

513 37. Sakata, R. C. et al. Base editors for simultaneous introduction of C-to-T and A-to-G

514 mutations. Nat. Biotechnol. 38, 865-869 (2020). 
Figdldre preprint doi: https://doi.org/10.1101/2022.03.04.482947; this version posted March 5, 2022. The copyright holder for this preprint (which was not certified by peer review) is the author/funder, who has granted bioRxiv a license to display the preprint in perpetuity. It is made available under aCC-BY 4.0 International license.

a

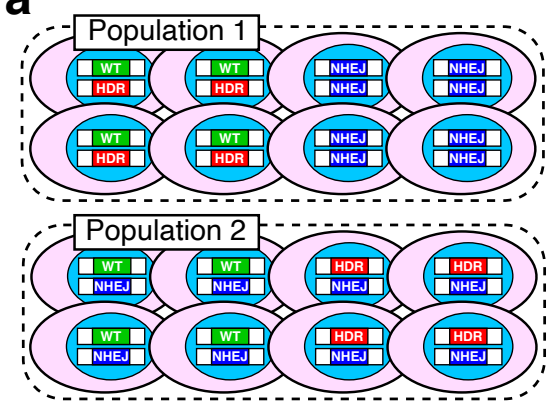

b

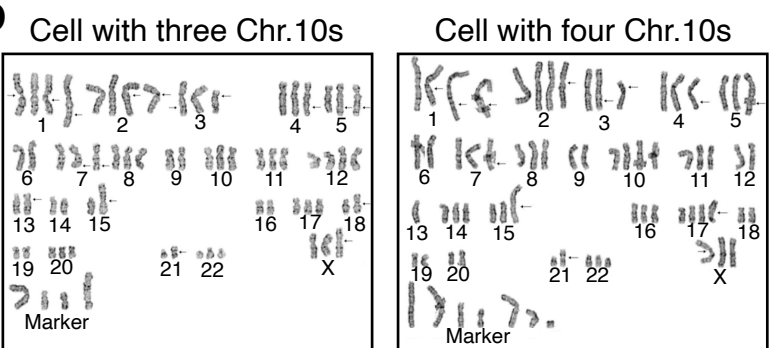

C

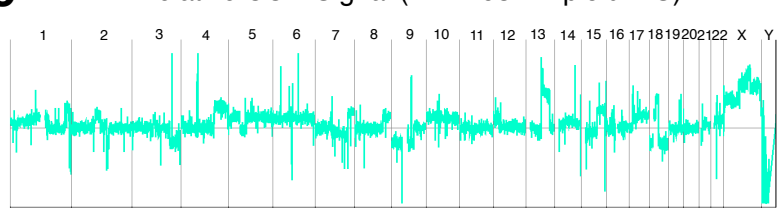

d

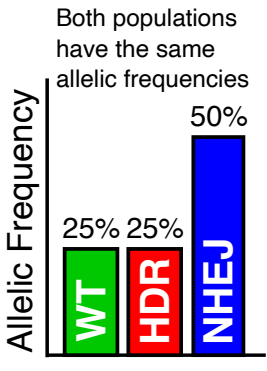

d CGH Copy Number Peak Assignment

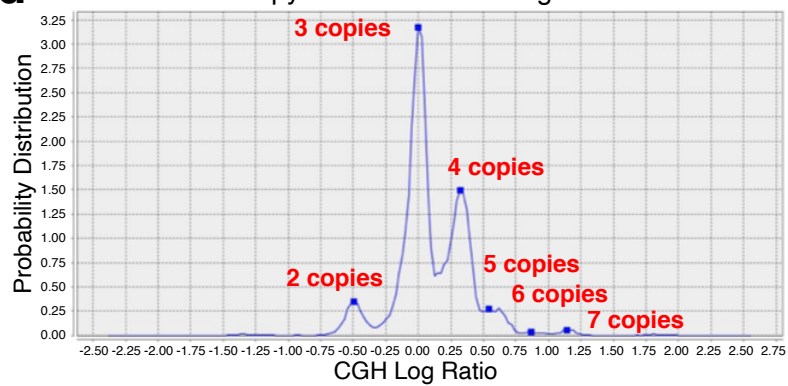

e

Relation of Copy Number and CGH Log Ratio

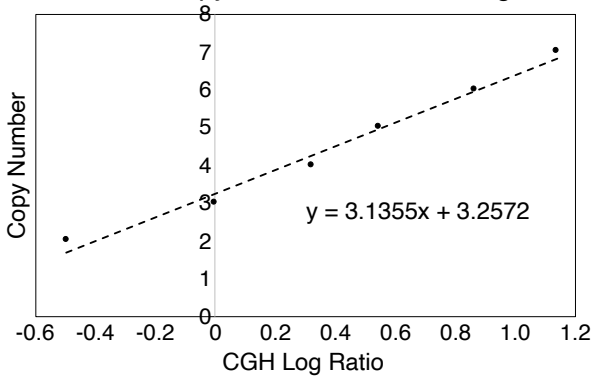

Scattered Plot of Relative CGH Signal (HEK293T/Diploid iPS) Around RBM20

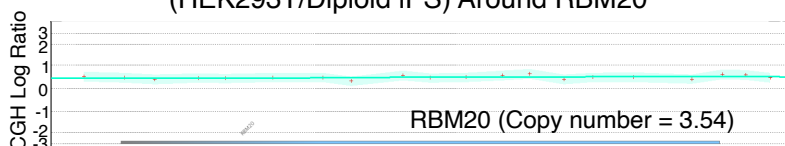

Figure 1. Measurement of the copy number of RBM20 in HEK293T cells.

(a) A hypothetical situation that emphasizes the importance of the analysis of genome editing outcomes at the single cell level. Cell populations 1 and 2 consist of cells with totally different genotypes individually. However, the total allelic frequencies of WT, HDR, and NHEJ are exactly the same for both populations. (b) Representative karyotypes of HEK293T cells with three and four chromosome 10s. (c) A CGH analysis of HEK293T cells in comparison to diploid human iPS cells. The relative CGH signal of HEK293T cells normalized to that of diploid iPS cells is shown throughout the genome. (d) CGH copy number peak assignment. In the CGH analysis, there were several peaks of the CGH signal ratio between HEK293T cells and diploid iPS cells based on the chromosomal numbers. The highest peak corresponded to three copies per cell, and was set as the baseline of the CGH log ratio between HEK293T cells and iPS cells. (e) Line of fit between the copy number and the CGH log ratio based on the peak assignment shown in (d). The copy number and the CGH log ratio showed a clear linear correlation. (f) Scattered plot of relative CGH signal of HEK293T cells in comparison to diploid human iPS cells around the RBM20 gene. The relative CGH signals of HEK293T cells normalized by that of iPS cells are represented by + . No microduplications or microdeletions were detected around the RBM20 locus. 


\section{Figure 2}

bioRxiv preprint doi: https://doi.org/10.1101/2022.03.04.482947; this version posted March 5, 2022. The copyright holder for this preprint (which was not certified by peer review) is the author/funder, who has granted bioRxiv a license to display the preprint in perpetuity. It is made available under aCC-BY 4.0 International license.

a WT Allele RBM20 Exon 9

ACAGATATGGCCCAGAAAGGCCGCGGTCT GTAGTCCGGTGAGCCGGTCACTCTCCCCGA TGTCTATACCGGGTCTTTCCGGCGCCAGAGCATCAGGCCACTCGGCCAGTGAGAGGGGCT (1)

Single-Stranded Oligo DNA Dono

ACAGATATGGCCCAGAAAGGCCGCGTCTAGTAGTCCGGTGAGCCGGTCACTCTCCCCGA

HDR Allele

ACAGATATGGCCCAGAAAGGCCGGGTCTAGTAGTCCGGTGAGCCGGTCACTCTCCCCGA TGTCTATACCGGGTCTTTCCGGCGCCAGATCATCAGGCCACTCGGCCAGTGAGAGGGGT

NHEJ Alleles

OR

ACAGATATGGCCCAGAAAGGCCGCGGTCTCGTAGTCCG-------CGGTCACTCTCCCCGA TGTCTATACCGGGTCTTTCCGGCGCCAGAGCATCAGGC------GCCAGTGAGAGGGGCT

ACAGATATGGCCCAGAAAGGCCGCGGTCTCATGAGTCCGGTGAAAAGCCGGTCACTCTCCCCGA TGTCTATACCGGGTCTTTCCGGCGCCAGAGTCATCAGGCCACTTTTCGGCCAGTGAGAGGGGT

HDR+NHEJ Allele

OR

ACAGATATGGCCCAGAAAGGCCGCGGTCTAGTAGTCCG------CGGTCACTCTCCCCGA TGTCTATACCGGGTCTTTCCGGCGCCAGATCATCAGGC-------GCCAGTGAGAGGGGCT

ACAGATATGGCCCAGAAAGGCCGCGGTCTAGTAGTCCGGTGAAAAGCCGGTCACTCTCCCCGA TGTCTATACCGGGTCTTTCCGGCGCCAGATCATCAGGCCACTTTTCGGCCAGTGAGAGGGGT

C
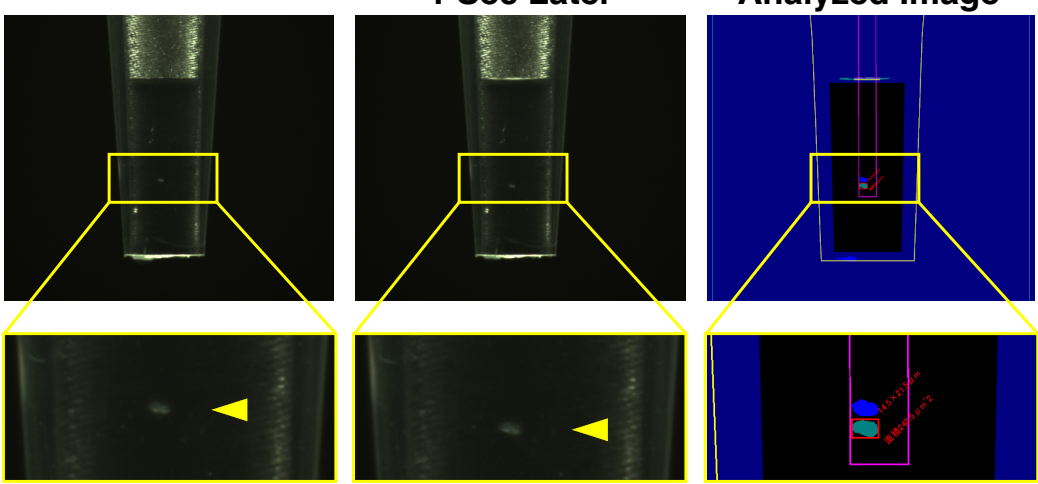

b
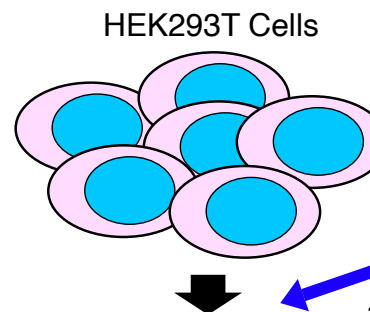

Transduction of Cas9-T2A-EGFP, gRNA, and donor DNA
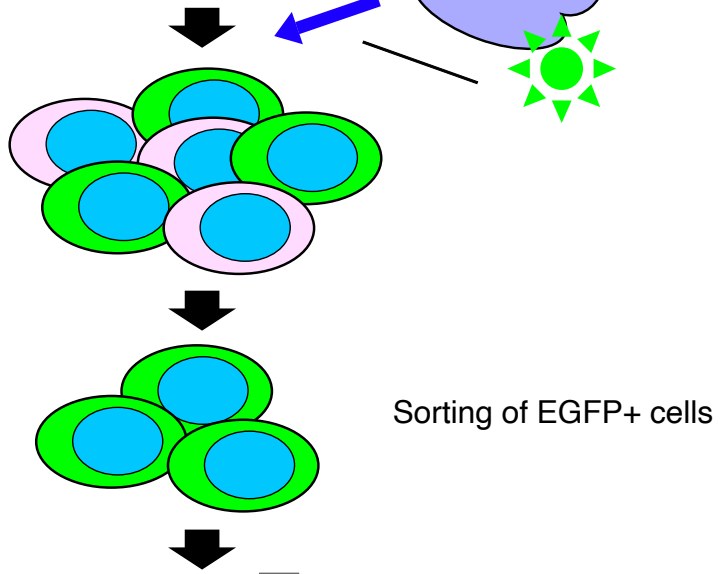

Single cell dispensing by SPiS

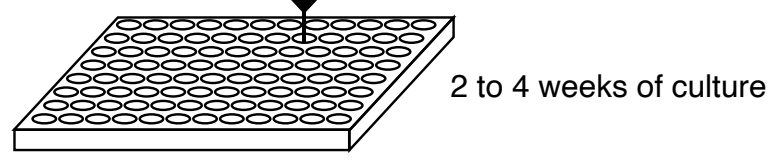

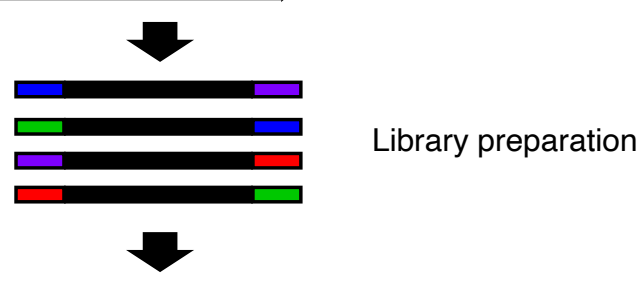

ATGCGTGTG . .

ATGCGTGTG...

ATGAATTGA ...

Amplicon-seq

\section{Figure 2. Schematic illustration of the experiments of this study.}

(a) Design of genome editing in RBM20 as a model case in this study. We introduced the R636S (c.1906C $>A$ ) mutation using CRISPR-Cas9 and a single-stranded oligonucleotide donor DNA. The resulting HDR allele has a $C$ to $A$ single nucleotide substitution, whereas the NHEJ alleles have various insertions and deletions. (b) The experimental flow of this study. We expressed Cas9-T2A-EGFP together with the gRNA targeting RBM20 to label HEK293T cells in which Cas9 protein was expressed. Using a microfluidic cell sorter (Onchip Sort), we sorted EGFP+ cells. Then, the sorted cells were plated into four 96-well plates by the SPiS. Cells were cultured for 2 to 4 weeks and then the genome editing outcomes were analyzed by amplicon sequencing. (c) An imaging analysis of single cells dispensed by the SPiS. The SPiS aspirates cell suspension into a specialized microtip and takes two images with a one-second interval. Only when the SPiS recognized a single cell, the content of the tip was dispensed into a well of a 96-well plate. Otherwise, the SPiS would take another aliquot of the cell suspension to repeat the process. 
Figddre proprint doi: https://doi.org/10.1101/2022.03.04.482947; this version posted March 5, 2022. The copyright holder for this preprint (which was not certified by peer review) is the author/funder, who has granted bioRxiv a license to display the preprint in perpetuity. It is made

a available under aCC-BY 4.0 International license. Allelic Frequencies in Individual Clones

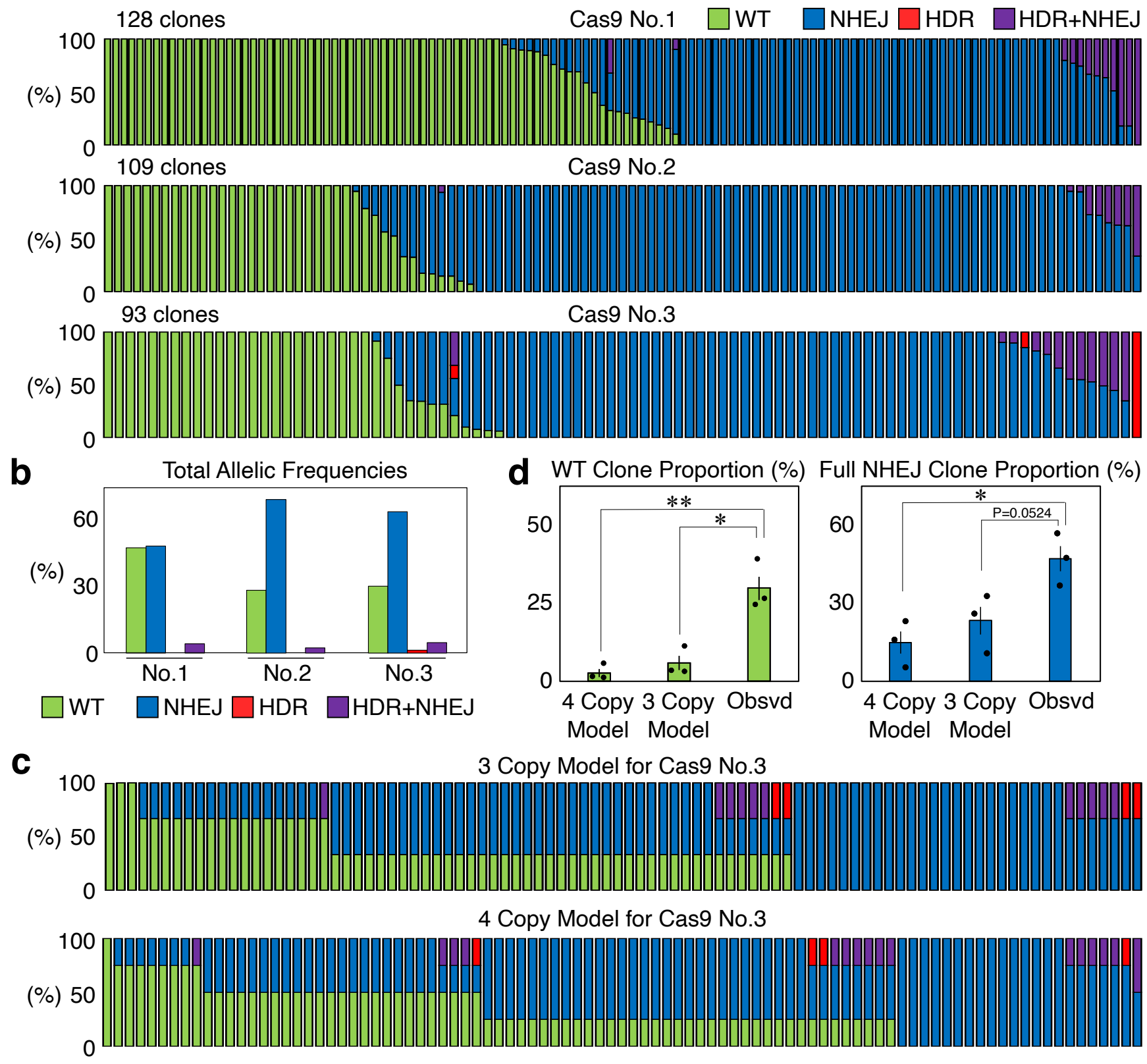

Figure 3. Genome editing outcomes in RBM20 in individual HEK293T cells edited by Cas9.

(a) Genome editing outcomes in isolated clones derived from single HEK293T cells edited by Cas9. We repeated the experiment three times, and isolated more than 90 clones out of 384 cells plated in all three trials. Each bar represents one clone, and the proportions of WT (green), NHEJ (blue), HDR (red), and HDR+NHEJ (purple) in one clone are also shown in each bar. (b) Total allelic frequencies in genome edited HEK293T cells in the three experiments shown in (a). (c) Models of the distributions of HEK293T cell clones with different genome editing outcomes in the Cas9 No.3 experiment, if genome editing randomly occurred at the frequencies shown in (b) in HEK293T cells with three or four copies of RBM20. (d) Comparison of the proportions of WT and full NHEJ clones between the mathematical models with three and four copies of RBM20 and the actually observed cells. Values \pm S.E. are shown $(n=3)$. Student's t-test was used to evaluate differences. ${ }^{*} \mathrm{P}<0.05$ and ${ }^{\star *} \mathrm{P}<0.01$. 
Figddrepreftrint doi: https://doi.org/10.1101/2022.03.04.482947; this version posted March 5, 2022. The copyright holder for this preprint (which was not certified by peer review) is the author/funder, who has granted bioRxiv a license to display the preprint in perpetuity. It is made available under aCC-BY 4.0 International license.

\section{Allelic Frequencies in Individual Clones}

a

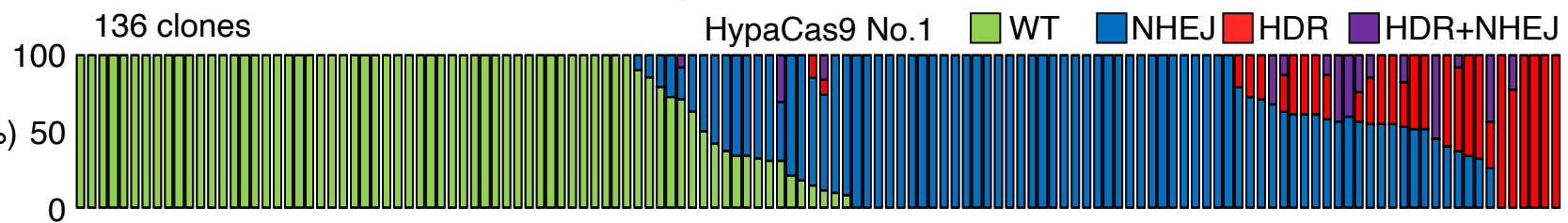

126 clones HypaCas9 No.2

(\%) 50

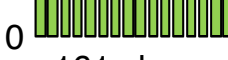

HypaCas9 No.3

(\%) 50

161 clones

H.
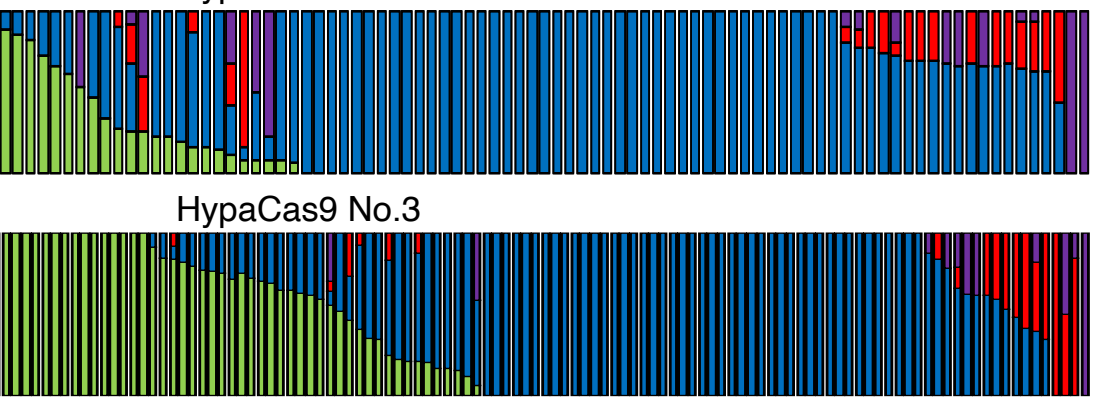

b

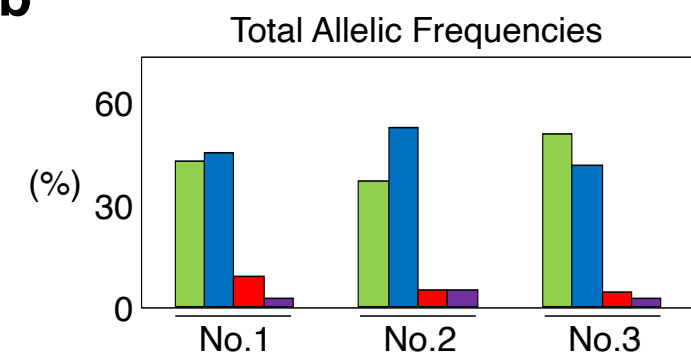

C

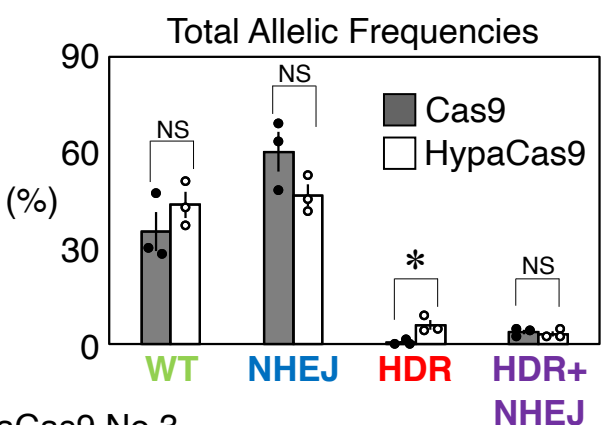

NHEJ

d

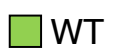

$\square$ NHEJ

$\square$ HDR

$\square$ HDR+NHEJ

3 Copy Model for HypaCas9 No.3

(\%) 50

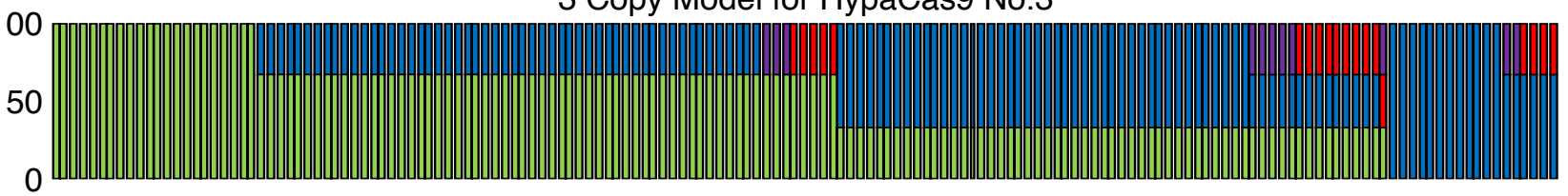

4 Copy Model for HypaCas9 No.3

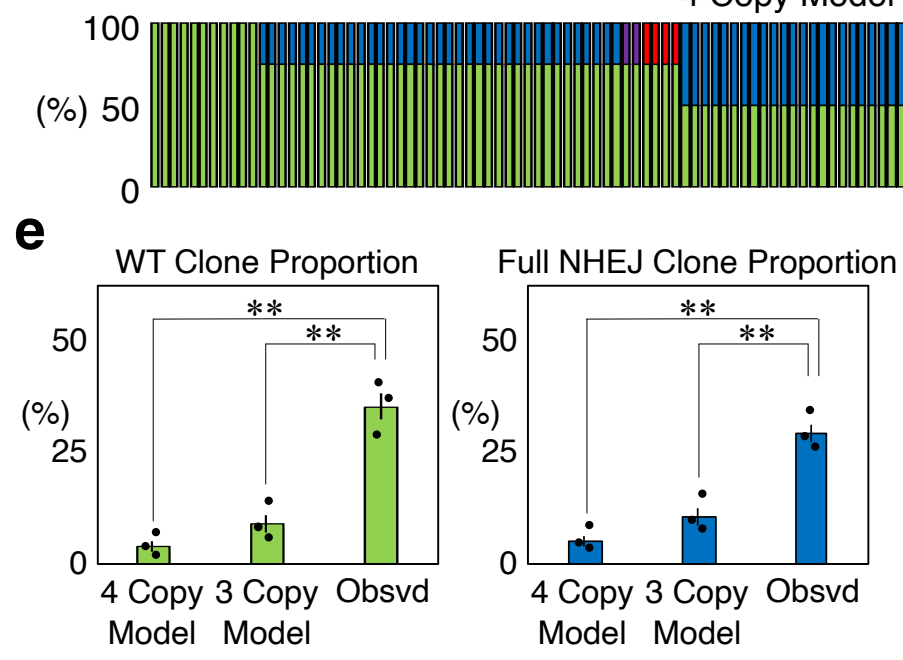

f

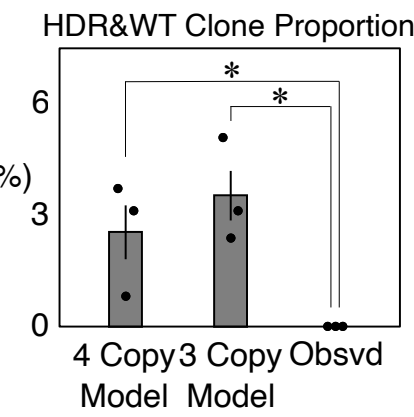

HDR Accomped by NHEJ

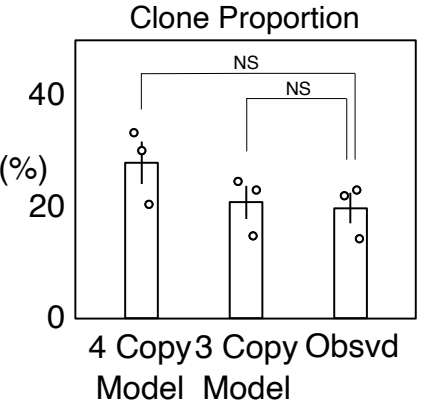




\section{HypaCas9.}

(a) Genome editing outcomes in isolated clones derived from single HEK293T cells edited by HypaCas9 and the single-stranded donor DNA. We repeated the same experiment three times. Each bar represents one clone, and the proportions of WT (green), NHEJ (blue), HDR (red), and HDR+NHEJ (purple) in one clone are also shown in each bar. (b) Total frequencies of WT (green), NHEJ (blue), HDR (red), and HDR+NHEJ (purple) allelic frequencies in genome edited HEK293T cells in the three experiments shown in (a). (c) Comparison of total allelic frequencies of WT, NHEJ, HDR, and HDR+NHEJ between Cas9 and HypaCas9. Values \pm S.E. are shown $(n=3)$. Student's $t$-test was used to evaluate differences. ${ }^{*} \mathrm{P}<0.05$ and NS: not significantly different $(P>0.1)$. (d) Models of the distributions of HEK293T cell clones with different genome editing outcomes in the HypaCas9 No.3 experiment, if genome editing randomly occurred at the frequencies shown in (b) in HEK293T cells with three or four copies of RBM20. (e) Comparison of the proportions of WT and full NHEJ clones between the mathematical models with three and four copies of RBM20 and the actually observed cells. Values \pm S.E. are shown $(n=3)$. Student's $t$-test was used to evaluate differences. ${ }^{*} \mathrm{P}<0.01$. (f) Comparison of the proportions of clones with partial editing by HDR and clones with HDR accompanied by NHEJ events between the models with three and four copies of RBM20 and the actually observed cells. Values \pm S.E. are shown $(n=3)$. Student's $t$-test was used to evaluate differences. ${ }^{*} \mathrm{P}<0.05$ and NS: not significantly different $(P>0.1)$. 
Figden proprint doi: https://doi.org/10.1101/2022.03.04.482947; this version posted March 5, 2022. The copyright holder for this preprint (which was not certified by peer review) is the author/funder, who has granted bioRxiv a license to display the preprint in perpetuity. It is made

a available under aCC-BY 4.0 International license. Allelic Frequencies in Individual Clones in ATP7B R778L Mutagenesis

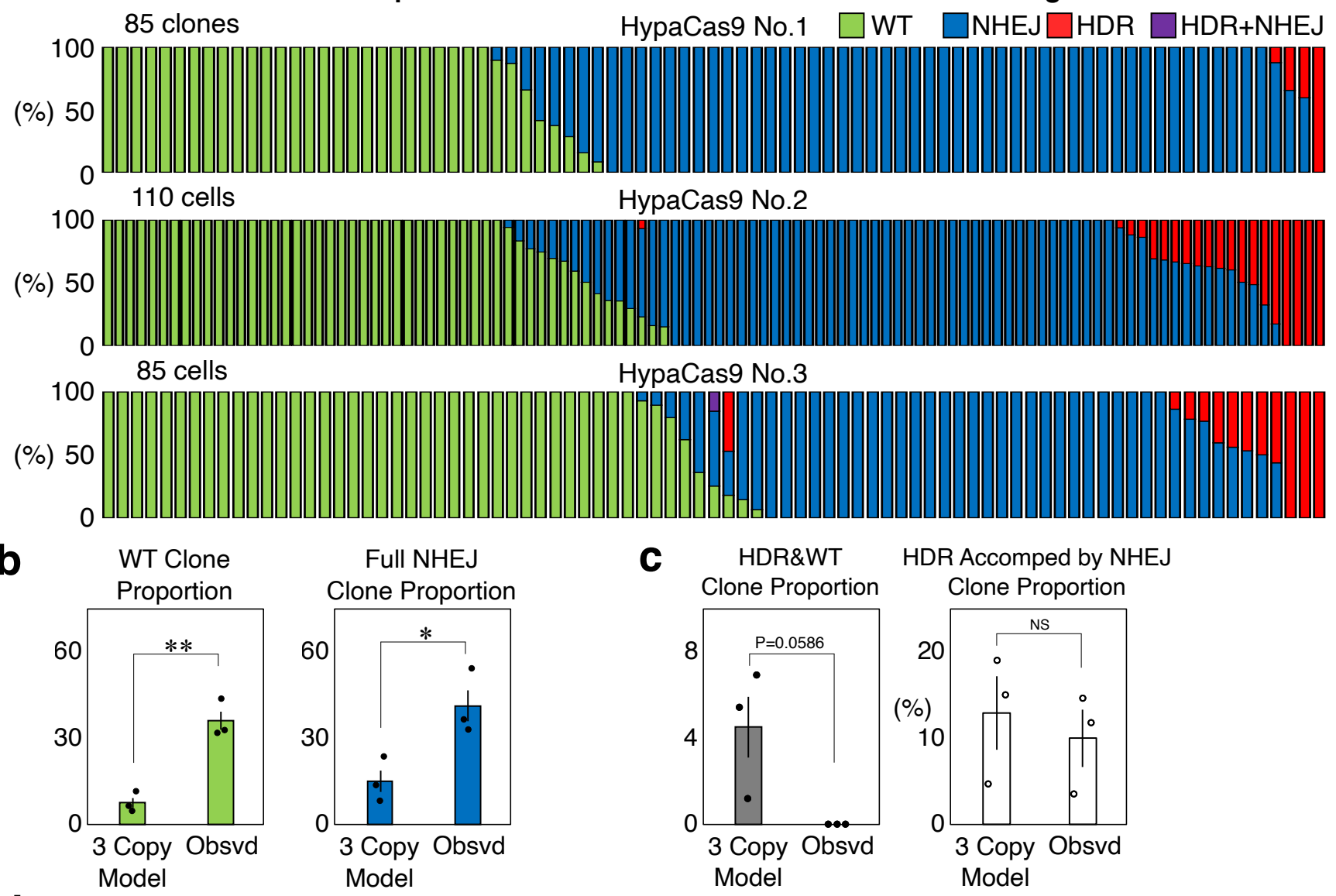

d

Allelic Frequencies in Individual Clones in GRN R493X Mutagenesis

84 clones

HypaCas9 No.1 $\square$ WT $\square$ NHEJ $\square$ HDR $\square$ HDR+NHEJ

(\%) 50
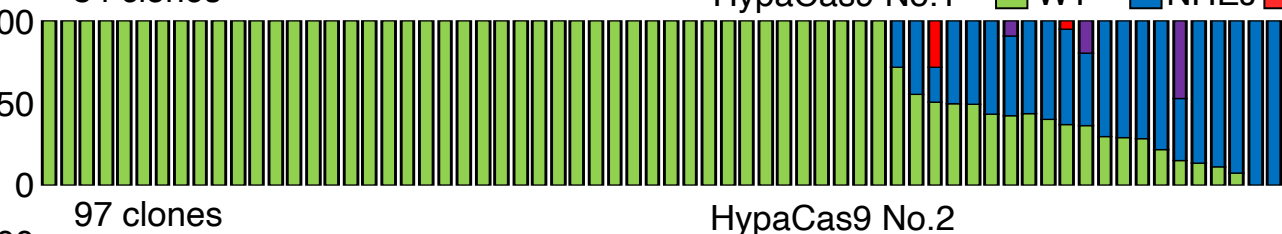

HypaCas9 No.2

(\%) 50

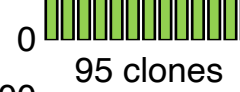

HypaCas9 No.3

(\%) 50

0

e

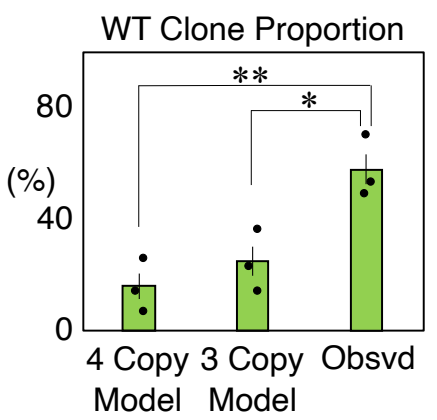

Full NHEJ Clone Proportion

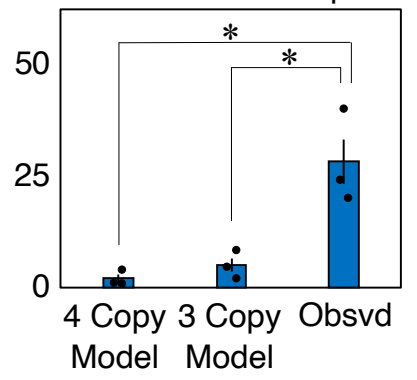




\section{edited by HypaCas9.}

(a) Genome editing outcomes in isolated clones derived from single HEK293T cells edited by HypaCas9 and the single-stranded donor DNA targeting ATP7B. We repeated the same experiment three times. Each bar represents one clone, and the proportions of WT (green), NHEJ (blue), HDR (red), and HDR+NHEJ (purple) in one clone are also shown in each bar. (b) Comparison of the proportions of WT and full NHEJ clones between the mathematical models with three copies of ATP7B and the actually observed cells.

Values \pm S.E. are shown $(n=3)$. Student's $t$-test was used to evaluate differences. ${ }^{*} P<0.05$ and ${ }^{* *} \mathrm{P}<0.01$. (c) Comparison of the proportions of clones with partial editing by HDR and clones with HDR accompanied by NHEJ events between the models with three copies of ATP7B and the actually observed cells. Values \pm S.E. are shown $(n=3)$. Student's $t$-test was used to evaluate differences. NS: not significantly different $(P>0.1)$. (d) Genome editing outcomes in isolated clones derived from single HEK293T cells edited by HypaCas9 and the single-stranded donor DNA targeting GRN. We repeated the same experiment three times. Each bar represents one clone, and proportions of WT (green), NHEJ (blue), HDR (red), and HDR+NHEJ (purple) in one clone are also shown in each bar. (e) Comparison of the proportions of WT and full NHEJ clones between the mathematical models with three and four copies of GRN and the actually observed cells. Values \pm S.E. are shown $(n=3)$. Student's $t$-test was used to evaluate differences. ${ }^{*} P<0.05$ and ${ }^{* *} \mathrm{P}<0.01$. 


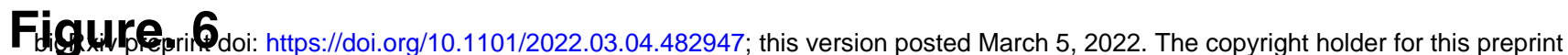
(which was not certified by peer review) is the author/funder, who has granted bioRxiv a license to display the preprint in perpetuity. It is made

a

Allelic Frequencies in Individual Clones in RBM20 R636S Mutagenesis

47 clones HypaCas9 No.1

(\%) 50

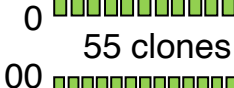

HypaCas9 No.2

(\%) 50

(1)

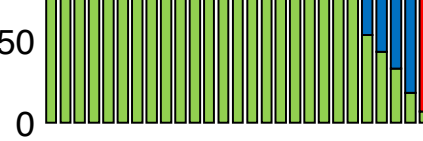

68 clones HypaCas9 No.3

(\%) 50

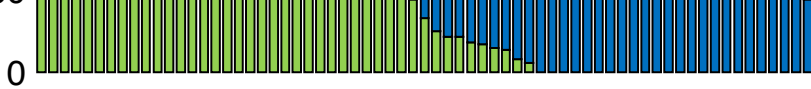

$\square$ WT $\square$ NHEJ $\square$ HDR $\square$ HDR+NHEJ

C

Allelic Frequencies in Individual Clones in ATP7B R778L Mutagenesis

63 clones HypaCas9 No.1

(\%) 50 100 on

(\%) 50

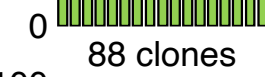

HypaCas9 No.2

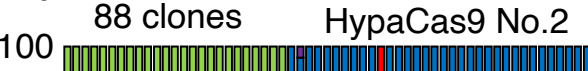<smiles>[Co]</smiles>

83 clones

HypaCas9 No.3

(\%) 50

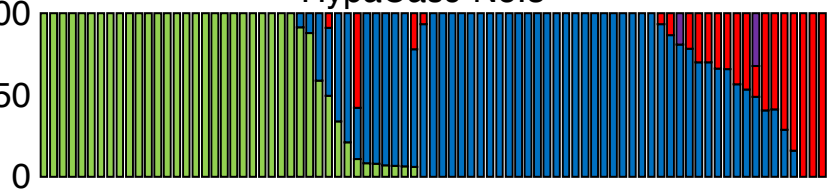

$\square$ WT $\square$ NHEJ $\square$ HDR $\square$ HDR+NHEJ

f

Allelic Frequencies in Individual Clones in GRN R493X Mutagenesis

51 clones HypaCas9 No.1

(\%) 50
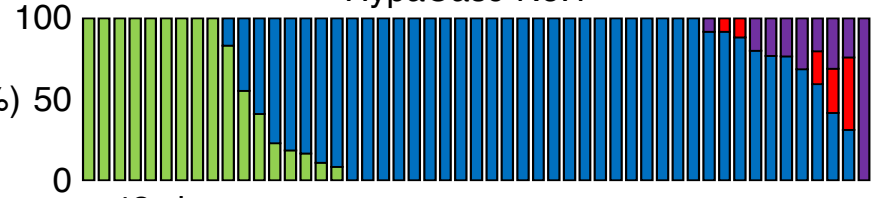
42 clones
HypaCas9 No.2

(\%) 50
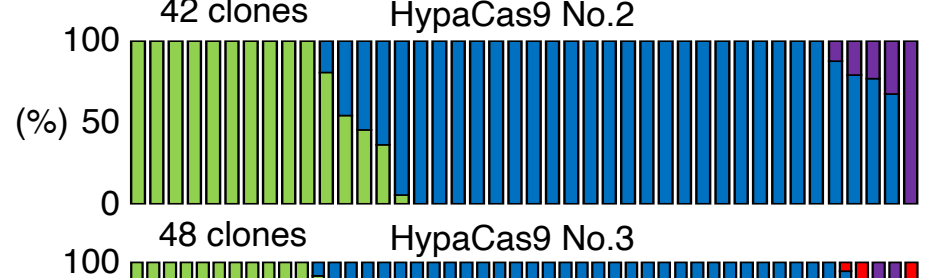

(\%) 50

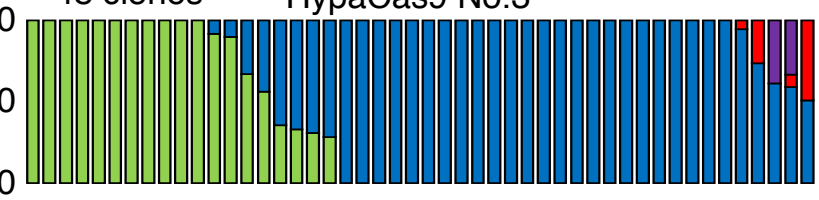

$\square$ WT $\square$ NHEJ $\square$ HDR $\square$ HDR+NHEJ

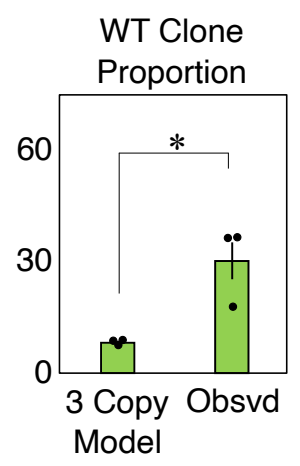

Full NHEJ

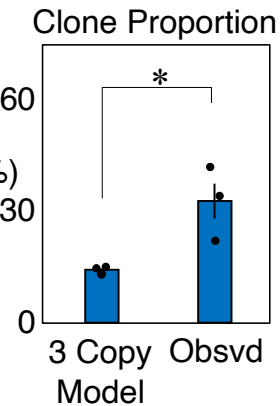

Full NHEJ

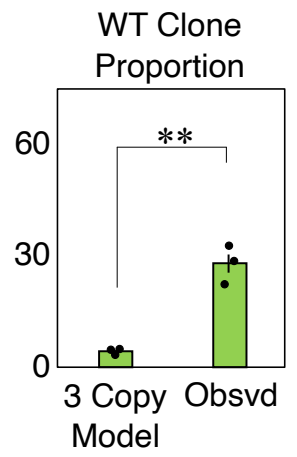

HDR\&WT
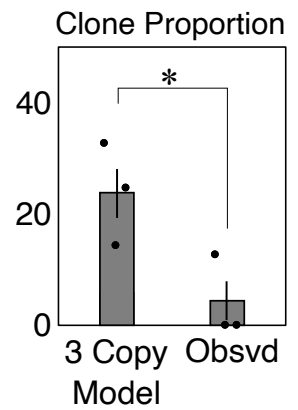

$(\%)$

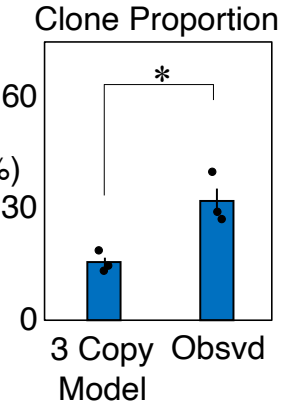

HDR Accomped by NHEJ

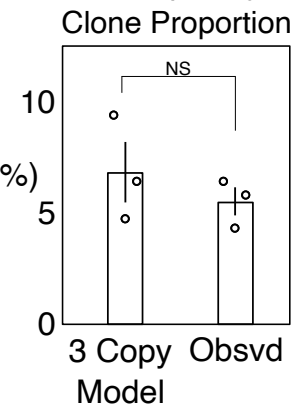

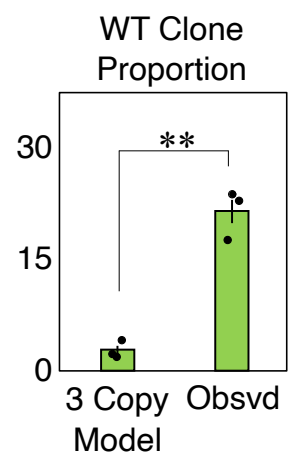

Full NHEJ

Clone Proportion

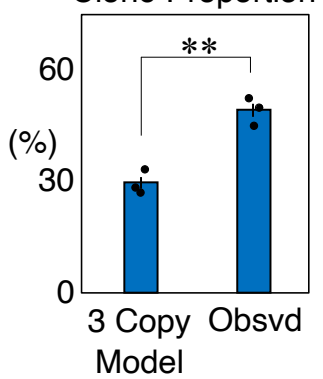


(a) Genome editing outcomes in isolated clones derived from single HeLa cells edited by HypaCas9 and the single-stranded donor DNA targeting RBM20. Each bar represents one clone, and the proportions of WT (green), NHEJ (blue), HDR (red), and HDR+NHEJ (purple) in one clone are also shown in each bar. (b) Comparison of the proportions of WT and full NHEJ clones between the mathematical models with three copies of RBM20 and the actually observed cells. Values \pm S.E. are shown $(n=3)$. Student's $t$-test was used to evaluate differences. ${ }^{\star} P<0.05$. (c) Genome editing outcomes in isolated clones derived from single HeLa cells edited by HypaCas9 and the single-stranded donor DNA targeting ATP7B. Each bar represents one clone, and the proportions of WT (green), NHEJ (blue), HDR (red), and HDR+NHEJ (purple) in one clone are also shown in each bar. (d) Comparison of the proportions of WT and full NHEJ clones between the mathematical models with three copies of ATP7B and the actually observed cells. Values \pm S.E. are shown $(n=3)$. Student's $t$-test was used to evaluate differences. ${ }^{*} P<0.05$ and ${ }^{* *} P<0.01$. (e) Comparison of the proportions of clones with partial editing by HDR and clones with HDR accompanied by NHEJ events between the models with three copies of ATP7B and the actually observed cells. Values \pm S.E. are shown $(n=3)$. Student's $t$-test was used to evaluate differences. ${ }^{*} \mathrm{P}<0.05$. NS: not significantly different $(P>0.1)$. (f) Genome editing outcomes in isolated clones derived from single HeLa cells edited by HypaCas 9 and the single-stranded donor DNA targeting GRN. Each bar represents one clone, and the proportions of WT (green), NHEJ (blue), HDR (red), and HDR+NHEJ (purple) in one clone are also shown in each bar. (g) Comparison of the proportions of WT and full NHEJ clones between the mathematical models with three copies of GRN and the actually observed cells. Values \pm S.E. are shown $(n=3)$. Student's t-test was used to evaluate differences. ${ }^{* *} \mathrm{P}<0.01$ 


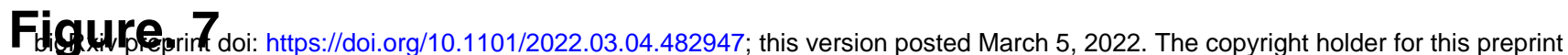
(which was not certified by peer review) is the author/funder, who has granted bioRxiv a license to display the preprint in perpetuity. It is made

a

Allelic Frequencies in Individual Clones in RBM20 R636S Mutagenesis

96 clones HypaCas9 No.1

(\%) 50

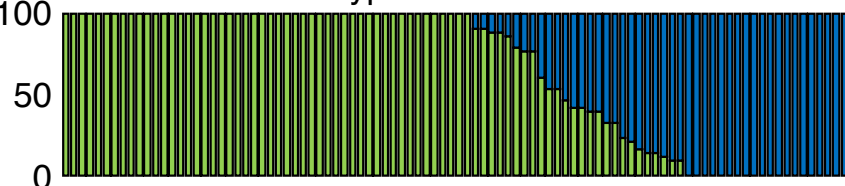

111 clones

HypaCas9 No.2

(\%) 50
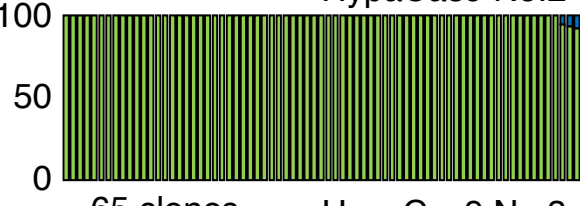

65 clones

HypaCas9 No.3

(\%) 50

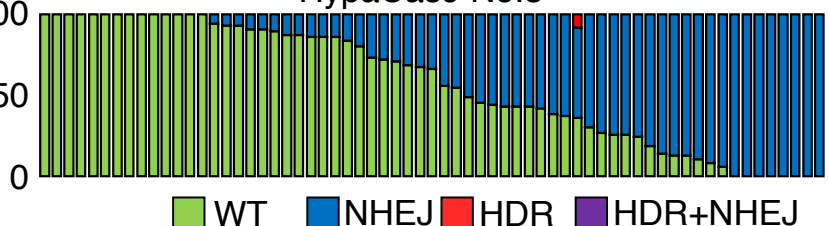

C

Allelic Frequencies in Individual Clones in ATP7B R778L Mutagenesis

74 clones HypaCas9 No.1

(\%) 50

100
50

0 clones HypaCas9 No.2
86 .

(\%) 50

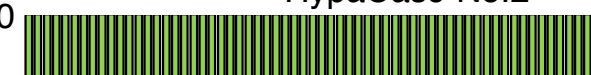

0

121 clones

HypaCas9 No.3

(\%) 50

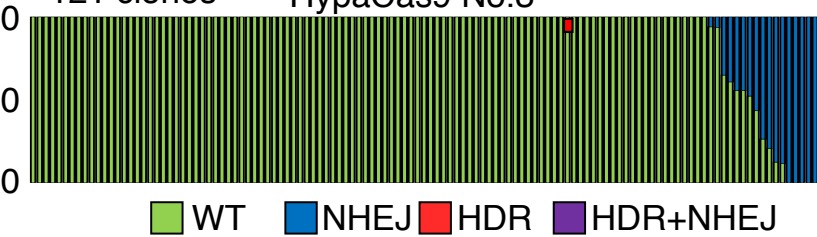

e

Allelic Frequencies in Individual Clones in GRN R493X Mutagenesis

76 cells HypaCas9 No.1

(\%) 50

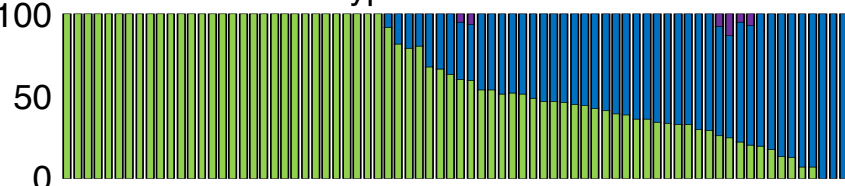

106 cells

HypaCas9 No.2

(\%) 50

100
50

96 cells

HypaCas9 No.3

(\%) 50

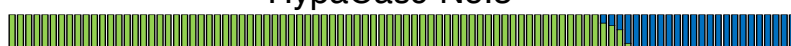

0

WT

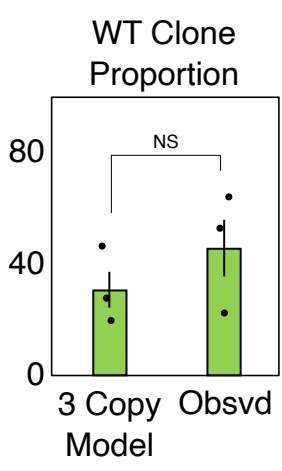

Full NHEJ

Clone Proportion

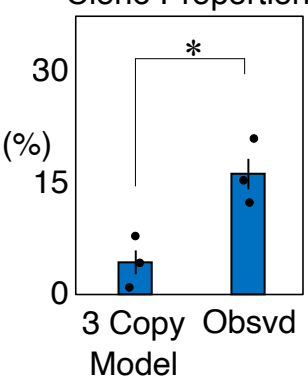

Full NHEJ

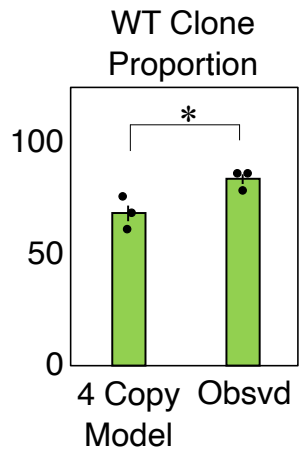

Clone Proportion

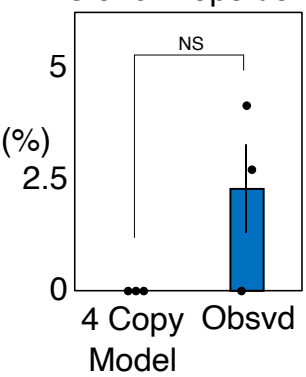

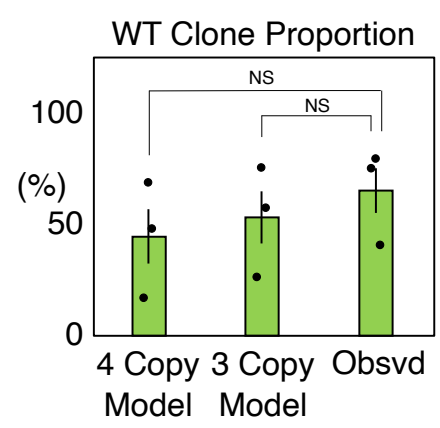

Full NHEJ Clone Proportion

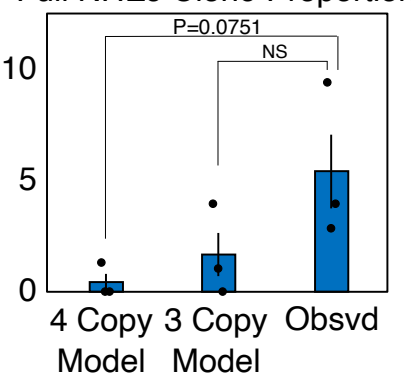


(a) Genome editing outcomes in isolated clones derived from single PC9 cells edited by HypaCas9 and the single-stranded donor DNA targeting RBM20. Each bar represents one clone, and the proportions of WT (green), NHEJ (blue), HDR (red), and HDR+NHEJ (purple) in one clone are also shown in each bar. (b) Comparison of the proportions of WT and full NHEJ clones between the mathematical models with three copies of RBM20 and the actually observed cells. Values \pm S.E. are shown $(n=3)$. Student's $t$-test was used to evaluate the difference. ${ }^{*} \mathrm{P}<0.05$. NS: not significantly different $(P>0.1)$. (c) Genome editing outcomes in isolated clones derived from single PC9 cells edited by HypaCas9 and the single-stranded donor DNA targeting ATP7B. Each bar represents one clone, and the proportions of WT (green), NHEJ (blue), HDR (red), and HDR+NHEJ (purple) in one clone are also shown in each bar. (d) Comparison of the proportions of WT and full NHEJ clones between the mathematical models with three copies of ATP7B and the actually observed cells. Values \pm S.E. are shown $(n=3)$. Student's $t$-test was used to evaluate differences. ${ }^{\star} \mathrm{P}<0.05$. NS: not significantly different $(P>0.1)$. (e) Genome editing outcomes in isolated clones derived from single PC9 cells edited by HypaCas9 and the single-stranded donor DNA targeting GRN. Each bar represents one clone, and the proportions of WT (green), NHEJ (blue), HDR (red), and HDR+NHEJ (purple) in one clone are also shown in each bar. (f) Comparison of the proportions of WT and full NHEJ clones between the mathematical models with three copies of GRN and the actually observed cells. Values \pm S.E. are shown $(n=3)$. Student's $t$-test was used to evaluate differences. NS: not significantly different $(P>0.1)$. 